\title{
Synthesis and antiviral activity evaluation of some new cyclohexylidenehydrazide derivatives of 1,3-thiazole core
}

\author{
Nuray ULUSOY GÜZELDEMİRCİ, Erkan PEHLIVAN, Zeynep HALAMOĞLU, Ayşe KOCABALKANLI
}

\section{ABSTRACT}

Six novel cyclohexylidenehydrazide derivatives carrying five membered heterocyclic ring, 1,3-thiazole were synthesized to investigate their antiviral (including anti-HIV) activity against diverse DNA and RNA viruses in CRFK, HeLa, HEL, MDCK,
Vero and MT4 cell cultures. None of the compounds was found active against any of the DNA or RNA viruses at $100 \mu \mathrm{M}$.

Keywords: 1,3-Thiazole, cyclohexylidenehydrazide, antiviral activity
Nuray Ulusoy Güzeldemirci, Erkan Pehlivan, Zeynep Halamoğlu, Ayşe Kocabalkanlı

Istanbul University, Faculty of Pharmacy, Department of Pharmaceutical Chemistry, 34116 Istanbul, Turkey

Corresponding author:

Nuray Ulusoy Güzeldemirci

Phone: +902124400000

Telefax: +902124400252

E-mail: nulusoy@istanbul.edu.tr

Submitted/Gönderilme: 18.03.2016 Accepted/Kabul: 15..04.2016
Revised/Düzeltme: 13.04.2016

\section{INTRODUCTION}

Viruses are the most common cause of global infectious disease. Viruses with high infection rates and rapid propagation can cause worldwide human and animal pandemics. Despite significant advances especially in antiviral therapy, there is continued interest in developing new agents for the treatment of viral diseases. The thiazole ring system is commonly found in many pharmaceutically important molecules. Numerous natural products containing this heterocycle have been isolated and exhibit significant biological activities (1). Thiazole plays vital roles in many drug structures. Ritonavir (anti-HIV drug) (2), Dasatinib and Tiazofurin (antineoplastic agents) (3), Fanetizole, Fentiazac and Meloxicam (anti-inflammatory agents) (4), Nizatidine (antiulcer agent) (5), Ravuconazole (antifungal agent) (6), Nitazoxanide (antiparasitic agent) (7) are some examples of thiazole bearing products (Figure 1) (8).

On the other hand, several hydrazide-ketone hydrazone derivatives have been reported to possess varios biological activities (9) such as, antiviral (10), antineoplastic (11), antituberculostatic $(12,13)$, analgesic (14), antibacterial (15), antifungal (16) activities.

In view of these observations, we report here the synthesis, structural determination and antiviral evaluation of new six $N^{2}$-substituted cyclohexylidene-2-(2-aminothiazol-4-yl) acetohydrazides. 
<smiles></smiles><smiles>NC(=O)c1csc(C2OC(CO)C(O)C2O)n1</smiles>

Tiazofurin

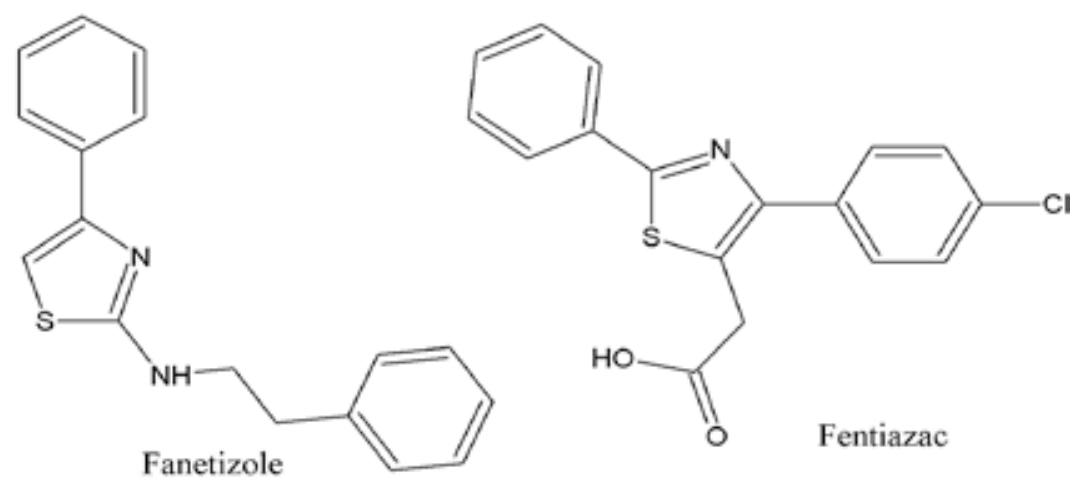<smiles>Cc1cnc(NC(=O)C2=C(O)c3ccccc3S(=O)(=O)N2C)s1</smiles>

Meloxicam<smiles>CN/C(=C\[N+](=O)[O-])NCCSCc1csc(CN(C)C)n1</smiles>

Nizatidine<smiles>[R4]O[R4]O[Na]</smiles><smiles>CC(=O)Oc1ccccc1C(=O)Nc1ncc([N+](=O)[O-])s1</smiles>

Nitazoxanide

Figure 1. Examples of thiazole bearing drugs. 


\section{MATERIALS AND METHODS}

\section{Chemistry}

Melting points were determined by using a Büchi B-540 melting point apparatus in open capillary tubes and are uncorrected. Elemental analyses were performed on a Thermo Finnigan Flash EA 1112 elemental analyzer. IR spectra were recorded on $\mathrm{KBr}$ discs, using a Shimadzu IR Affinity-1 FT-IR spectrophotometer. ${ }^{1} \mathrm{H}-\mathrm{NMR}$ spectra were measured on a Varian UNITY INOVA $(500 \mathrm{MHz})$ spectrometer using $D M S O-\mathrm{d}_{6}$. The starting materials were commercially available.

\section{Preparation of 2-(2-aminothiazol-4-yl)acetohydrazide (2)}

To a solution of ethyl 2-(2-aminothiazol-4-yl)acetate (1) $(0.005 \mathrm{~mol})$ in ethanol $(30 \mathrm{~mL})$ were added hydrazine hydrate $(0.025 \mathrm{~mol}, 98 \%)$. The reaction mixture was refluxed for $5 \mathrm{~h}$ then cooled and allowed to stand overnight. The crystals were filtered, dried and purified by crystallization from ethanol.

\section{General procedure for the synthesis of $N^{2}$-(substituted} cyclohexylidene)-2-(2-aminothiazol-4-yl)acetohydrazides (3a-f)

A solution of substituted cyclohexanones $(0.005 \mathrm{~mol})$ and 2-(2-aminothiazol-4-yl)acetohydrazide (2) $(0.005 \mathrm{~mol})$ in absolute ethanol $(30 \mathrm{ml})$ was refluxed for $6 \mathrm{~h}$ and allowed to stand overnight. The crystals thus obtained were filtered, then recrystallized from ethanol.

$\mathrm{N}^{2}$-(3-methylcyclohexylidene)-2-(2-aminothiazol-4-yl) acetohydrazide (3a)

Yield 80\%; mp: 191-192 ${ }^{\circ}$; IR $(\mathrm{KBr}) v_{\max }\left(\mathrm{cm}^{-1}\right): 3277(\mathrm{~N}-$ $\mathrm{H}), 1685(\mathrm{C}=\mathrm{O}) .{ }^{1} \mathrm{H}-\mathrm{NMR} \quad\left(\mathrm{DMSO}_{\mathrm{d}}\right) \delta(\mathrm{ppm})$ : 0.92; $0.95\left(2 \mathrm{~d}, 3 \mathrm{H}, J=5.86 ; 6.35 \mathrm{~Hz}, \mathrm{CH}_{3}\right), 1.13-1.17(\mathrm{~m}, 1 \mathrm{H}$, cyclohexylidene $\left.\mathrm{C}_{3}-\mathrm{H}\right), 1.38-1.42(\mathrm{~m}, 1 \mathrm{H}$, cyclohexylidene $\left.\mathrm{C}_{5 \text {-axial }} \mathrm{H}\right), 1.53-1.61\left(\mathrm{~m}, 2 \mathrm{H}\right.$, cyclohexylidene $\mathrm{C}_{4 \text {-axial }} \mathrm{H}$ ve $\mathrm{C}_{5 \text { - }}$ equatorial $\mathrm{H}), 1.69-1.71\left(\mathrm{~m}, 1 \mathrm{H}\right.$, cyclohexylidene $\left.\mathrm{C}_{2 \text {-axial }} \mathrm{H}\right), 1.80$ $1.84\left(\mathrm{~m}, 1 \mathrm{H}\right.$, cyclohexylidene $\left.\mathrm{C}_{\text {4-equatorial }} \mathrm{H}\right), 2.04-2.10(\mathrm{~m}, 1 \mathrm{H}$, cyclohexylidene $\left.\mathrm{C}_{6 \text {-axial }} \mathrm{H}\right), 2.25-2.27(\mathrm{~m}, 1 \mathrm{H}$, cyclohexylidene $\left.\mathrm{C}_{\text {6-equatorial }} \mathrm{H}\right), 2.76 ; 2.87(2 \mathrm{~d}, 1 \mathrm{H}, J=10.73 \mathrm{~Hz}$, cyclohexylidene $\left.\mathrm{C}_{\text {2-equatorial }} \mathrm{H}\right), 3.36 ; 3.68\left(2 \mathrm{~s}, 2 \mathrm{H}, \mathrm{CH}_{2} \mathrm{CO}\right), 6.18 ; 6.25(2 \mathrm{~s}, 1 \mathrm{H}$, thiazole $\left.\mathrm{C}_{5}-\mathrm{H}\right), 6.75 ; 6.86\left(2 \mathrm{~s}, 2 \mathrm{H}, \mathrm{NH}_{2}\right), 10.17 ; 10.28(2 \mathrm{~s}, 1 \mathrm{H}$, CONH). Anal. Calcd. for $\mathrm{C}_{12} \mathrm{H}_{18} \mathrm{~N}_{4}$ OS: C, 54.11; H, 6.81; N, 21.03; Found: C, 54.02; H, 6.56; N, 20.97.

$\mathrm{N}^{2}$-(4-methylcyclohexylidene)-2-(2-aminothiazol-4-yl) acetohydrazide (3b)

Yield 73\%; mp: 188-189 ${ }^{\circ}$; IR $(\mathrm{KBr}) v_{\max }\left(\mathrm{cm}^{-1}\right): 3367(\mathrm{~N}-$ $\mathrm{H}), 1653(\mathrm{C}=\mathrm{O}) .{ }^{1} \mathrm{H}-\mathrm{NMR}\left(\right.$ DMSO-d $\left._{6}\right) \delta(\mathrm{ppm}): 0.91(\mathrm{~d}, 3 \mathrm{H}$, $\left.J=6.34 \mathrm{~Hz}, \mathrm{CH}_{3}\right), 1.00-1.15\left(\mathrm{~m}, 2 \mathrm{H}\right.$, cyclohexylidene $\mathrm{C}_{4}-\mathrm{H}$ ve $\left.\mathrm{C}_{3 \text {-axial }} \mathrm{H} / \mathrm{C}_{5 \text {-axial }} \mathrm{H}\right), 1.62-1.69(\mathrm{~m}, 1 \mathrm{H}$, cyclohexylidene $\left.\mathrm{C}_{3 \text {-axial }} \mathrm{H} / \mathrm{C}_{5 \text {-axial }} \mathrm{H}\right), 1.74-1.91 \quad\left(\mathrm{~m}, 3 \mathrm{H}\right.$, cyclohexylidene $\mathrm{C}_{3-}$ equatorial $\mathrm{H}, \mathrm{C}_{5 \text {-equatorial }} \mathrm{H}$ ve $\left.\mathrm{C}_{2 \text {-axial }} \mathrm{H} / \mathrm{C}_{6 \text {-xxial }} \mathrm{H}\right), 2.13-2.21$ (m,
$1 \mathrm{H}$, cyclohexylidene $\left.\mathrm{C}_{2 \text {-axial }} \mathrm{H} / \mathrm{C}_{6 \text {-axial }} \mathrm{H}\right), 2.25-2.32(\mathrm{~m}, 1 \mathrm{H}$, cyclohexylidene $\left.\mathrm{C}_{\text {2-equatorial }} \mathrm{H} / \mathrm{C}_{\text {6-equatorial }} \mathrm{H}\right), 2.81 ; 2.92(2 \mathrm{~d}, 1 \mathrm{H}$, $J=14.64 \mathrm{~Hz}$, cyclohexylidene $\left.\mathrm{C}_{2 \text {-equatorial }} \mathrm{H} / \mathrm{C}_{6 \text {-equatorial }} \mathrm{H}\right), 3.35$; $3.68\left(2 \mathrm{~s}, 2 \mathrm{H}, \mathrm{CH}_{2} \mathrm{CO}\right), 6.19 ; 6.25\left(2 \mathrm{~s}, 1 \mathrm{H}\right.$, thiazole $\left.\mathrm{C}_{5}-\mathrm{H}\right)$, $6.78 ; 6.90\left(2 \mathrm{~s}, 2 \mathrm{H}, \mathrm{NH}_{2}\right), 10.21 ; 10.32(2 \mathrm{~s}, 1 \mathrm{H}, \mathrm{CONH})$. Anal. Calcd. for $\mathrm{C}_{12} \mathrm{H}_{18} \mathrm{~N}_{4}$ OS: C, 54.11; H, 6.81; N, 21.03; Found: C, 53.19; H, 7.03; N, 21.25.

$\mathrm{N}^{2}$-(4-ethylcyclohexylidene)-2-(2-aminothiazol-4-yl) acetohydrazide (3c)

Yield 74\%; mp: $181-182^{\circ} \mathrm{C}$; IR $(\mathrm{KBr}) \nu_{\max }\left(\mathrm{cm}^{-1}\right): 3373(\mathrm{~N}-$ $\mathrm{H}), 1656(\mathrm{C}=\mathrm{O}) .{ }^{1} \mathrm{H}-\mathrm{NMR}\left(\mathrm{DMSO}-\mathrm{d}_{6}\right) \delta(\mathrm{ppm}): 0.87(\mathrm{t}, 3 \mathrm{H}$, $\left.J=7.32 \mathrm{~Hz}, \mathrm{CH}_{3}\right), 0.93-1.12\left(\mathrm{~m}, 2 \mathrm{H}\right.$, cyclohexylidene $\mathrm{C}_{4}-\mathrm{H}$ ve $\left.\mathrm{C}_{3 \text {-axial }} \mathrm{H} / \mathrm{C}_{5-\text {-axial }} \mathrm{H}\right), 1.20-1.27\left(\mathrm{~m}, 2 \mathrm{H}, \mathrm{CH}_{2}\right), 1.40-1.44(\mathrm{~m}$, $1 \mathrm{H}$, cyclohexylidene $\left.\mathrm{C}_{3 \text {-axial }} \mathrm{H} / \mathrm{C}_{5 \text {-axial }} \mathrm{H}\right), 1.77-1.90(\mathrm{~m}, 3 \mathrm{H}$, cyclohexylidene $\mathrm{C}_{3 \text {-equatorial }} \mathrm{H}, \mathrm{C}_{5 \text {-equatorial }} \mathrm{H}$ ve $\mathrm{C}_{2 \text {-axial }} \mathrm{H} / \mathrm{C}_{6 \text {-axial }} \mathrm{H}$ ), 2.12-2.19 (m, $1 \mathrm{H}$, cyclohexylidene $\left.\mathrm{C}_{\text {2-axial }} \mathrm{H} / \mathrm{C}_{6 \text {-axial }} \mathrm{H}\right), 2.27$ $2.33\left(\mathrm{~m}, 1 \mathrm{H}\right.$, cyclohexylidene $\left.\mathrm{C}_{2 \text {-equatorial }} \mathrm{H} / \mathrm{C}_{6 \text {-equatorial }} \mathrm{H}\right), 2.82$; $2.93\left(2 \mathrm{~d}, 1 \mathrm{H}, J=15.61 ; 14.15 \mathrm{~Hz}\right.$, cyclohexylidene $\mathrm{C}_{\text {2-equatorial }} \mathrm{H} /$ $\left.\mathrm{C}_{6 \text {-equatorial }} \mathrm{H}\right), 3.43 ; 3.69\left(2 \mathrm{~s}, 2 \mathrm{H}, \mathrm{CH}_{2} \mathrm{CO}\right), 6.19 ; 6.25(2 \mathrm{~s}, 1 \mathrm{H}$, thiazole $\left.\mathrm{C}_{5}-\mathrm{H}\right), 6.78 ; 6.90\left(2 \mathrm{~s}, 2 \mathrm{H}, \mathrm{NH}_{2}\right), 10.20 ; 10.33(2 \mathrm{~s}, 1 \mathrm{H}$, CONH). Anal. Calcd. for $\mathrm{C}_{13} \mathrm{H}_{20} \mathrm{~N}_{4}$ OS: C, 55.69; H, 7.19; N, 19.98; Found: C, 55.09; H, 7.75; N, 19.97.

$\mathrm{N}^{2}$-(4-propylcyclohexylidene)-2-(2-aminothiazol-4-yl) acetohydrazide (3d)

Yield 39\%; mp: 179-180 ${ }^{\circ} \mathrm{C}$; IR $(\mathrm{KBr}) v_{\max }\left(\mathrm{cm}^{-1}\right): 3369(\mathrm{~N}-\mathrm{H})$, $1658(\mathrm{C}=\mathrm{O}) .{ }^{1} \mathrm{H}-\mathrm{NMR}\left(\mathrm{DMSO}-\mathrm{d}_{6}\right) \delta(\mathrm{ppm}): 0.88(\mathrm{t}, 3 \mathrm{H}, J=7.32$ $\left.\mathrm{Hz}, \mathrm{CH}_{3}\right), 0.96-1.12\left(\mathrm{~m}, 2 \mathrm{H}\right.$, cyclohexylidene $\mathrm{C}_{4}-\mathrm{H}_{\text {ve }} \mathrm{C}_{3 \text {-axial }} \mathrm{H} /$ $\left.\mathrm{C}_{5 \text {-axial }} \mathrm{H}\right), 1.17-1.23\left(\mathrm{~m}, 2 \mathrm{H}\right.$, cyclohexylidene 4- $\left.\mathrm{CH}_{2} \mathrm{CH}_{2} \mathrm{CH}_{3}\right)$, 1.26-1.33 (m, 2H, cyclohexylidene 4- $\mathrm{CH}_{2} \mathrm{C}_{2} \mathrm{CH}_{3}$ ), 1.49$1.55\left(\mathrm{~m}, 1 \mathrm{H}\right.$, cyclohexylidene $\left.\mathrm{C}_{3 \text {-axial }} \mathrm{H} / \mathrm{C}_{5 \text {-axial }} \mathrm{H}\right), 1.78-1.90(\mathrm{~m}$, $3 \mathrm{H}$, cyclohexylidene $\mathrm{C}_{3 \text {-equatorial }} \mathrm{H}, \mathrm{C}_{5 \text {-equatorial }} \mathrm{H}$ ve $\mathrm{C}_{2 \text {-axial }} \mathrm{H} / \mathrm{C}_{6}$ $\left.{ }_{\text {axial }} \mathrm{H}\right), 2.12-2.20\left(\mathrm{~m}, 1 \mathrm{H}\right.$, cyclohexylidene $\left.\mathrm{C}_{2 \text {-axial }} \mathrm{H} / \mathrm{C}_{6 \text {-axial }} \mathrm{H}\right)$, 2.26-2.33 (m, $1 \mathrm{H}$, cyclohexylidene $\left.\mathrm{C}_{\text {2-equatorial }} \mathrm{H} / \mathrm{C}_{6 \text {-equatorial }} \mathrm{H}\right)$, 2.82; $2.93\left(2 \mathrm{~d}, 1 \mathrm{H}, J=15.13 ; 15.62 \mathrm{~Hz}\right.$, cyclohexylidene $\mathrm{C}_{2}$. equatorial $\left.\mathrm{H} / \mathrm{C}_{\text {-equatorial }} \mathrm{H}\right), 3.35 ; 3.68\left(2 \mathrm{~s}, 2 \mathrm{H}, \mathrm{CH}_{2} \mathrm{CO}\right), 6.18 ; 6.25$ $\left(2 \mathrm{~s}, 1 \mathrm{H}\right.$, thiazole $\left.\mathrm{C}_{5}-\mathrm{H}\right), 6.78 ; 6.90\left(2 \mathrm{~s}, 2 \mathrm{H}, \mathrm{NH}_{2}\right), 10.20 ; 10.32$ (2s, $1 \mathrm{H}, \mathrm{CONH})$. Anal. Calcd. for $\mathrm{C}_{14} \mathrm{H}_{22} \mathrm{~N}_{4}$ OS: C, 57.11; $\mathrm{H}$, 7.53; N, 19.03; Found: C, 56.20; H, 7.61; N, 19.58 .

$\mathrm{N}^{2}$-(4-tert-butylcyclohexylidene)-2-(2-aminothiazol-4-yl) acetohydrazide (3e)

Yield 82\%; mp: 157-158 $\mathrm{C}$; IR (KBr) $v_{\max }\left(\mathrm{cm}^{-1}\right): 3402$ $(\mathrm{N}-\mathrm{H}), 1660(\mathrm{C}=\mathrm{O}) .{ }^{1} \mathrm{H}-\mathrm{NMR}\left(\mathrm{DMSO}-\mathrm{d}_{6}\right) \delta(\mathrm{ppm}): 0.85$ (s, 9H, cyclohexylidene 4- $\left.\mathrm{C}\left(\mathrm{CH}_{3}\right)_{3}\right), 1.04-1.15(\mathrm{~m}, 2 \mathrm{H}$, cyclohexylidene $\mathrm{C}_{4}-\mathrm{H}$ ve $\mathrm{C}_{3 \text {-axial }} \mathrm{H} / \mathrm{C}_{5 \text {-xxial }} \mathrm{H}$ ), 1.26-1.29 (m, $1 \mathrm{H}$, cyclohexylidene $\left.\mathrm{C}_{3 \text {-axial }} \mathrm{H} / \mathrm{C}_{5 \text {-axial }} \mathrm{H}\right), 1.79-1.90(\mathrm{~m}, 3 \mathrm{H}$, cyclohexylidene $\mathrm{C}_{3 \text {-equatorial }} \mathrm{H}, \mathrm{C}_{5 \text {-equatorial }} \mathrm{H}$ ve $\mathrm{C}_{2 \text {-axial }} \mathrm{H} / \mathrm{C}_{6 \text {-axial }} \mathrm{H}$ ), 2.12-2.16 $\left(\mathrm{m}, 1 \mathrm{H}\right.$, cyclohexylidene $\left.\mathrm{C}_{\text {2-axial }} \mathrm{H} / \mathrm{C}_{6 \text {-axial }} \mathrm{H}\right), 2.34$ - 
$2.37\left(\mathrm{~m}, 1 \mathrm{H}\right.$, cyclohexylidene $\left.\mathrm{C}_{\text {2-equatorial }} \mathrm{H} / \mathrm{C}_{6 \text {-equatorial }} \mathrm{H}\right), 2.89$; $3.02\left(2 \mathrm{~d}, 1 \mathrm{H}, J=14.64 \mathrm{~Hz}\right.$, cyclohexylidene $\mathrm{C}_{\text {2-equatorial }} \mathrm{H} / \mathrm{C}_{6-}$ $\left.{ }_{\mathrm{ial}} \mathrm{H}\right), 3.35 ; 3.68\left(2 \mathrm{~s}, 2 \mathrm{H}, \mathrm{CH}_{2} \mathrm{CO}\right), 6.18 ; 6.25(2 \mathrm{~s}, 1 \mathrm{H}$, thiazole $\left.\mathrm{C}_{5}-\mathrm{H}\right), 6.75 ; 6.88\left(2 \mathrm{~s}, 2 \mathrm{H}, \mathrm{NH}_{2}\right), 10.16 ; 10.31(2 \mathrm{~s}, 1 \mathrm{H}$, $\mathrm{CONH}$ ). Anal. Calcd. for $\mathrm{C}_{15} \mathrm{H}_{24} \mathrm{~N}_{4}$ OS: C, 58.41; H, 7.84; N, 18.16; Found: C, 58.26; H, 7.68; N, 18.12.

$N^{2}$-(4-phenylcyclohexylidene)-2-(2-aminothiazol-4-yl) acetohydrazide (3f)

Yield 91\%; mp: 209-210 ${ }^{\circ} \mathrm{C}$; IR (KBr) $v_{\max }\left(\mathrm{cm}^{-1}\right): 3365(\mathrm{~N}-$ $\mathrm{H}), 1658(\mathrm{C}=\mathrm{O}) .{ }^{1} \mathrm{H}-\mathrm{NMR}$ (DMSO-d $\left.{ }_{6}\right) \delta(\mathrm{ppm}): 1.51-1.59$ $\left(\mathrm{m}, 1 \mathrm{H}\right.$, cyclohexylidene $\left.\mathrm{C}_{3 \text {-axial }} \mathrm{H} / \mathrm{C}_{5 \text {-axial }} \mathrm{H}\right), 1.60-1.70(\mathrm{~m}$, $1 \mathrm{H}$, cyclohexylidene $\left.\mathrm{C}_{3 \text {-axial }} \mathrm{H} / \mathrm{C}_{5 \text {-axial }} \mathrm{H}\right), 1.90-2.05(\mathrm{~m}, 2 \mathrm{H}$, cyclohexylidene $\mathrm{C}_{3 \text {-equatorial }} \mathrm{H}$ ve $\left.\mathrm{C}_{5 \text {-equatorial }} \mathrm{H}\right), 2.32-2.49(\mathrm{~m}, 3 \mathrm{H}$, cyclohexylidene $\mathrm{C}_{2 \text {-axial }} \mathrm{H}, \mathrm{C}_{6 \text {-axial }} \mathrm{H}$ ve $\left.\mathrm{C}_{\text {2-equatorial }} \mathrm{H} / \mathrm{C}_{\text {6-equatorial }} \mathrm{H}\right)$, 2.82-2.87 (m, 1H, cyclohexylidene $\left.\mathrm{C}_{4}-\mathrm{H}\right), 3.01 ; 3.13(2 \mathrm{~d}, 1 \mathrm{H}$, $J=15.62 ; 15.61 \mathrm{~Hz}$, cyclohexylidene $\mathrm{C}_{\text {2-equatorial }} \mathrm{H} / \mathrm{C}_{6 \text {-equatorial }} \mathrm{H}$ ), $3.38 ; 3.73\left(2 \mathrm{~s}, 2 \mathrm{H}, \mathrm{CH}_{2} \mathrm{CO}\right), 6.22 ; 6.27\left(2 \mathrm{~s}, 1 \mathrm{H}\right.$, thiazole $\mathrm{C}_{5}$ $\mathrm{H}), 6.80 ; 6.91\left(2 \mathrm{~s}, 2 \mathrm{H}, \mathrm{NH}_{2}\right), 7.17-7.30$ (m, 5H, phenyl), $10.30 ; 10.41(2 \mathrm{~s}, 1 \mathrm{H}, \mathrm{CONH})$. Anal. Calcd. for $\mathrm{C}_{17} \mathrm{H}_{20} \mathrm{~N}_{4} \mathrm{OS}$ : C, 62.17; H, 6.14; N, 17.06; Found: C, 61.78; H, 6.26; N, 17.26.

\section{Biological activity}

\section{Antiviral evaluations in cell culture}

The compounds (3a-f) were evaluated for activity against diverse RNA- and DNA-viruses, using the following cellbased assays (17): (a) Crandell-Rees Feline Kidney (CRFK) cells infected with Feline corona virus or Feline herpes virus; (b) Human embryonic lung (HEL) fibroblast cells infected with Herpes simplex virus-1 or -2 , Vaccinia virus, Vesicular stomatitis virus, an Acyclovir-resistant herpes simplex virus-1 strain, or Adenovirus-2; (c) Human cervixcarcinoma HeLa cells infected with Vesicular stomatitis virus, Coxsackie B4 virus or Respiratory syncytial virus; (d) African green monkey kidney Vero cells infected with Para-influenza-3 virus, Reovirus-1, Sindbis virus, Coxsackie B4 virus, Punta toro virus or Yellow fever virus; (e) Madin-Darby canine kidney (MDCK) cells infected with Influenza $\mathrm{A} / \mathrm{H} 1 \mathrm{~N} 1$ subtype (A/Ned/378/05), Influenza A/H3N2 subtype (A/ $\mathrm{HK} / 7 / 87)$ or Influenza B (B/Ned/537/05); and (f) Human T-lymphoblast MT4- cells infected with HIV-1 or HIV-2.

To perform the antiviral assays, the virus was added to subconfluent cell cultures in 96-well plates, and at the same time, the test compounds were added at serial dilutions. Appropriate reference compounds were included, i.e. the virus entry inhibitor dextran sulfate 5000, the broad antiviral agent ribavirin, the antiherpetic drug ganciclovir, and the HIV inhibitor azidothymidine. After 3-6 days incubation at $37{ }^{\circ} \mathrm{C}$ (or $35^{\circ} \mathrm{C}$ in the case of influenza virus), the cultures were examined by microscopy to score the compounds' inhibitory effect on virus-induced cytopathic effect (CPE) or their cytotoxicity. For some viruses [Influenza A/H1N1 (A/ $\mathrm{Ned} / 378 / 05)$, Influenza A/H3N2 (A/HK/7/87) and Influenza $\mathrm{B}(\mathrm{B} / \mathrm{Ned} / 537 / 05)]$ antiviral and cytotoxic activities were confirmed by the colorimetric MTS cell viability assay.

\section{RESULTS AND DISCUSSION}

2-(2-Aminothiazol-4-yl)acetic acid hydrazide (2) was prepared from ethyl 2-(2-aminothiazol-4-yl)acetate (1) according to a previously published procedure (18). Condensation of 2 with substituted cyclohexanones afforded the corresponding $N^{2}$-substituted cyclohexylidene-2-(2-aminothiazol-4-yl) acetohydrazides (3a-f) (Scheme 1). The structures of the obtained compounds were elucidated by spectral data. The IR spectra of 3a-f showed two separate bands resulting from the $\mathrm{N}-\mathrm{H}$ and $\mathrm{C}=\mathrm{O}$ streching bands of the amide function at about regions 3402-3277 and 1685-1653 $\mathrm{cm}^{-1}$, respectively (19, 20). In the spectra of ${ }^{1} \mathrm{H}-\mathrm{NMR}$ of $\mathbf{3 a}-\mathbf{f}$ the $\mathrm{CH}_{2}$ and $\mathrm{CONH}$ of the acetylamino moiety were observed as a double singlets presumably due to the partial double bond character of the $\mathrm{C}-\mathrm{N}$ bond and the bulk of the attached cyclohexyl structure which can disrupt free rotation about the cited bond $(\delta 3.73$ 3.35 and $\delta 10.41-10.16 \mathrm{ppm}$, respectively) (21). The protons of the 1,3-thiazole and substituted cyclohexylidene nucleus resonated at the expected regions (22-25).

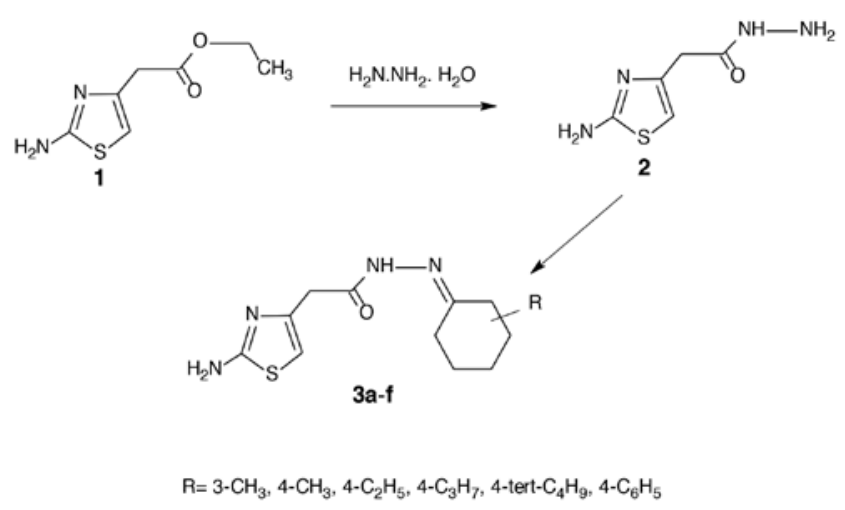

Scheme 1. The synthetic pathway of the title compounds (3a-f).

The synthesized compounds were evaluated against a broad and diverse panel of RNA- and DNA-viruses using cytopathic effect (CPE) reduction assays in appropriate cell culture models (17). As can be seen in Tables 1-6, none of the synthesized compounds was found active (i.e. minimal antivirally effective concentration $\geq 5$-fold lower than minimal cytotoxic concentration) against any of the DNA or RNA viruses, including HIV virus at $100 \mu \mathrm{M}$. 
Table 1. Anti-feline corona virus (FIPV) and anti-feline herpes virus activity and cytotoxicity of the compounds 3a-3f in CRFK cell cultures.

\begin{tabular}{|c|c|c|c|}
\hline \multirow[b]{2}{*}{ Compound } & \multirow[b]{2}{*}{$\mathrm{CC}_{50}{ }^{\mathrm{a}}(\mu \mathrm{M})$} & \multicolumn{2}{|c|}{$\mathrm{EC}_{50}{ }^{\mathrm{b}}(\mu \mathrm{M})$} \\
\hline & & $\begin{array}{l}\text { Feline Corona Virus } \\
\text { (FIPV) }\end{array}$ & Feline Herpes Virus \\
\hline $3 a$ & $>100$ & $>100$ & $>100$ \\
\hline $3 \mathbf{b}$ & $>100$ & $>100$ & $>100$ \\
\hline $3 c$ & $>100$ & $>100$ & $>100$ \\
\hline $3 d$ & $>100$ & $>100$ & $>100$ \\
\hline $3 e$ & $>100$ & $>100$ & $>100$ \\
\hline $3 f$ & $>100$ & $>100$ & $>100$ \\
\hline HHA $(\mu \mathrm{g} / \mathrm{ml})$ & $>100$ & 11 & 2.9 \\
\hline UDA $(\mu \mathrm{g} / \mathrm{ml})$ & $>100$ & 3.3 & 1.6 \\
\hline Ganciclovir $(\mu \mathrm{M})$ & $>100$ & $>100$ & 2.2 \\
\hline
\end{tabular}

${ }^{a} 50 \%$ Cytototoxic concentration, as determined by measuring the cell viability with the colorimetric formazan-based MTS assay.

${ }^{\mathrm{b}} 50 \%$ Effective concentration, or concentration producing $50 \%$ inhibition of virus-induced cytopathic effect, as determined by measuring the cell viability with the colorimetric formazan-based MTS assay.

Table 2. Antiviral activity and cytotoxicity of the compounds $\mathbf{3 a - 3 f}$ in HEL cell cultures.

\begin{tabular}{|c|c|c|c|c|c|c|c|}
\hline \multirow{2}{*}{ Compound } & \multirow{2}{*}{$\begin{array}{l}\mathrm{MCC}^{\mathrm{a}} \\
(\mu \mathrm{M})\end{array}$} & \multirow[b]{2}{*}{$\begin{array}{c}\text { Herpes } \\
\text { simplex } \\
\text { virus-1 } \\
\text { (KOS) }\end{array}$} & \multirow[b]{2}{*}{$\begin{array}{c}\text { Herpes } \\
\text { simplex } \\
\text { virus-2 }(G)\end{array}$} & \multirow[b]{2}{*}{$\begin{array}{c}\text { Herpes simplex } \\
\text { virus-1 TK- KOS } \\
\text { ACV }^{\mathrm{r}}\end{array}$} & \multicolumn{2}{|l|}{$\mathrm{EC}_{50}{ }^{\mathrm{b}}(\mu \mathrm{M})$} & \multirow[b]{2}{*}{$\begin{array}{c}\text { Vesicular } \\
\text { stomatitis } \\
\text { virus }\end{array}$} \\
\hline & & & & & $\begin{array}{c}\text { Vaccinia } \\
\text { virus }\end{array}$ & Adenovirus-2 & \\
\hline $3 \mathbf{a}$ & $>100$ & $>100$ & $>100$ & $>100$ & $>100$ & $>100$ & $>100$ \\
\hline $3 b$ & $>100$ & $>100$ & $>100$ & $>100$ & $>100$ & $>100$ & $>100$ \\
\hline $3 c$ & $>100$ & $>100$ & $>100$ & $>100$ & $>100$ & $>100$ & $>100$ \\
\hline $3 d$ & $>100$ & $>100$ & $>100$ & $>100$ & $>100$ & $>100$ & $>100$ \\
\hline $3 e$ & $>100$ & $>100$ & $>100$ & $>100$ & $>100$ & $>100$ & $>100$ \\
\hline $3 f$ & $>100$ & $>100$ & $>100$ & $>100$ & $>100$ & $>100$ & $>100$ \\
\hline Brivudin & $>250$ & 0.04 & 250 & $>250$ & 6.8 & - & $>250$ \\
\hline Cidofovir & $>250$ & 2.0 & 0.80 & 1.2 & 6.8 & 19 & $>250$ \\
\hline Acyclovir & $>250$ & 0.4 & 0.2 & 125 & $>250$ & - & $>250$ \\
\hline Ganciclovir & $>100$ & 0.06 & 0.03 & 4.0 & $>100$ & - & $>100$ \\
\hline Zalcitabine & $>250$ & - & - & - & - & 15 & - \\
\hline Alovudine & $>250$ & - & - & - & - & 22 & - \\
\hline
\end{tabular}

${ }^{a}$ Required to cause a microscopically detectable alteration of normal cell morphology.

${ }^{b}$ Required to reduce virus-induced cytopathogenicity by $50 \%$. 
Table 3. Antiviral activity and cytotoxicity of the compounds 3a-3f in HeLa cell cultures.

\begin{tabular}{|c|c|c|c|c|}
\hline & $\begin{array}{l}\text { Cytotoxicity } \\
\qquad(\mu \mathrm{M})\end{array}$ & & Antiviral $\mathrm{EC}_{50}{ }^{\mathrm{b}}(\mu \mathrm{M})$ & \\
\hline Compound & $\mathrm{MCC}^{\mathrm{a}}$ & $\begin{array}{l}\text { Vesicular stomatitis } \\
\text { virus }\end{array}$ & Coxsackie virus B4 & Respiratory syncytial virus \\
\hline $3 \mathbf{a}$ & $>100$ & $>100$ & $>100$ & $>100$ \\
\hline $3 b$ & $>100$ & $>100$ & $>100$ & $>100$ \\
\hline $3 c$ & $>100$ & $>100$ & $>100$ & $>100$ \\
\hline $3 d$ & $>100$ & $>100$ & $>100$ & $>100$ \\
\hline $3 e$ & $>100$ & $>100$ & $>100$ & $>100$ \\
\hline $3 f$ & $>100$ & $>100$ & $>100$ & $>100$ \\
\hline DS-10000 $(\mu \mathrm{g} / \mathrm{ml})$ & $>100$ & 0.8 & 34 & 0.4 \\
\hline Ribavirin $(\mu \mathrm{M})$ & $>250$ & 26 & 112 & 5.8 \\
\hline
\end{tabular}

${ }^{a}$ Required to cause a microscopically detectable alteration of normal cell morphology.

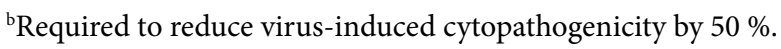

Table 4. Antiviral activity and cytotoxicity of the compounds $\mathbf{3 a - 3}$ f in Vero cell cultures.

\begin{tabular}{|c|c|c|c|c|c|c|c|}
\hline \multirow[b]{2}{*}{ Compound } & \multirow{2}{*}{$\begin{array}{c}\text { MCC }^{a} \\
(\mu \mathrm{M})\end{array}$} & \multicolumn{5}{|c|}{ Antiviral $\mathrm{EC}_{50}{ }^{\mathrm{b}}(\mu \mathrm{M})$} & \multirow[b]{2}{*}{$\begin{array}{c}\text { Yellow } \\
\text { Fever virus }\end{array}$} \\
\hline & & $\begin{array}{c}\text { Para-influenza-3 } \\
\text { virus }\end{array}$ & Reovirus-1 & $\begin{array}{l}\text { Sindbis } \\
\text { virus }\end{array}$ & $\begin{array}{l}\text { Coxsackie } \\
\text { virus B4 }\end{array}$ & Punta Toro virus & \\
\hline $3 \mathbf{a}$ & $>100$ & $>100$ & $>100$ & $>100$ & $>100$ & $>100$ & $>100$ \\
\hline $3 \mathbf{b}$ & $>100$ & $>100$ & $>100$ & $>100$ & $>100$ & $>100$ & $>100$ \\
\hline $3 c$ & $>100$ & $>100$ & $>100$ & $>100$ & $>100$ & $>100$ & $>100$ \\
\hline $3 d$ & $>100$ & $>100$ & $>100$ & $>100$ & $>100$ & $>100$ & $>100$ \\
\hline $3 e$ & $>100$ & $>100$ & $>100$ & $>100$ & $>100$ & $>100$ & $>100$ \\
\hline $3 f$ & $>100$ & $>100$ & $>100$ & $>100$ & $>100$ & $>100$ & $>100$ \\
\hline DS-10000 $(\mu \mathrm{M})$ & $>100$ & $>100$ & $>100$ & 4.0 & 58 & 100 & 0.60 \\
\hline Ribavirin $(\mu \mathrm{M})$ & $>250$ & 146 & 112 & $>250$ & $>250$ & 112 & 250 \\
\hline $\begin{array}{l}\text { Mycophenolic } \\
\text { acid }(\mu \mathrm{M})\end{array}$ & $>100$ & 0.8 & 2.0 & 4.0 & $>250$ & 10 & 1.8 \\
\hline
\end{tabular}

${ }^{a}$ Required to cause a microscopically detectable alteration of normal cell morphology.

${ }^{\mathrm{b}}$ Required to reduce virus-induced cytopathogenicity by $50 \%$. 
Table 5. Anti-influenza virus activity and cytotoxicity of compounds 3a-3f in MDCK cell cultures.

\begin{tabular}{|c|c|c|c|c|c|c|c|c|}
\hline \multirow[t]{2}{*}{ Compound } & \multicolumn{2}{|c|}{ Cytotoxicity $(\mu \mathrm{M})$} & \multicolumn{2}{|c|}{$\begin{array}{c}\text { Influenza A/H1N1 (A/ } \\
\text { Ned/378/05) }\end{array}$} & \multicolumn{2}{|c|}{$\begin{array}{c}\text { Influenza } A / H 3 N 2 \\
(A / H K / 7 / 87)^{c}\end{array}$} & \multicolumn{2}{|c|}{$\begin{array}{c}\text { Influenza B } \\
(B / \text { Ned/537/05) }\end{array}$} \\
\hline & $\mathrm{CC}_{50}{ }^{\mathrm{a}}$ & $\mathrm{MCC}^{\mathrm{b}}$ & CPE & MTS & CPE & MTS & CPE & MTS \\
\hline $3 \mathbf{a}$ & $>100$ & $>100$ & $>100$ & $>100$ & $>100$ & $>100$ & $>100$ & $>100$ \\
\hline $3 b$ & $>100$ & $>100$ & $>100$ & $>100$ & $>100$ & $>100$ & $>100$ & $>100$ \\
\hline $3 c$ & $>100$ & $>100$ & $>100$ & $>100$ & $>100$ & $>100$ & $>100$ & $>100$ \\
\hline $3 d$ & $>100$ & $>100$ & $>100$ & $>100$ & $>100$ & $>100$ & $>100$ & $>100$ \\
\hline $3 e$ & $>100$ & $>100$ & $>100$ & $>100$ & $>100$ & $>100$ & $>100$ & $>100$ \\
\hline $3 f$ & $>100$ & $>100$ & $>100$ & $>100$ & $>100$ & $>100$ & $>100$ & $>100$ \\
\hline Zanamivir & $>100$ & $>100$ & 0.8 & 0.4 & 0.5 & 1.5 & 0.4 & 0.8 \\
\hline Ribavirin & $>100$ & $>100$ & 8.9 & 7.0 & 8.9 & 7.6 & 2.6 & 6.7 \\
\hline Amantadine & $>200$ & $>200$ & 40 & 13.7 & 0.8 & 0.8 & $>200$ & $>200$ \\
\hline Rimantadine & $>200$ & $>200$ & 1.6 & 0.8 & 0.8 & 0.7 & $>200$ & $>200$ \\
\hline
\end{tabular}

a50\%Cytotoxic concentration, as determined by measuring the cell viability with the colorimetric formazan-based MTS assay.

${ }^{b}$ Minimum compound concentration that causes a microscopically detectable alteration of normal cell morphology.

${ }^{\mathrm{c}} \mathrm{EC}_{50}: 50 \%$ effective concentration or concentration producing $50 \%$ inhibition of virus-induced cytopathic effect, as determined by visual scoring of the cytopathic effect (CPE) or by measuring the cell viability with the colorimetric formazan-based MTS assay.

Table 6. Anti-HIV activity and cytotoxicity of the compounds 3a-3f in MT4 cellsa.

\begin{tabular}{|c|c|c|c|}
\hline \multirow{2}{*}{ Compound } & \multirow{2}{*}{$\begin{array}{l}\text { Cytotoxicity }(\mu \mathrm{M}) \\
\qquad \mathrm{CC}_{50}\end{array}$} & \multicolumn{2}{|c|}{ Antiviral EC $\mathrm{EC}_{50}(\mu \mathrm{M})$} \\
\hline & & HIV-1 (strain IIIB) & HIV-1 (strain ROD) \\
\hline $3 a$ & $>125$ & $>125$ & $>125$ \\
\hline $\mathbf{3 b}$ & $>125$ & $>125$ & $>125$ \\
\hline $3 c$ & $>125$ & $>125$ & $>125$ \\
\hline $3 d$ & $>125$ & $>125$ & $>125$ \\
\hline $3 e$ & $>125$ & $>125$ & $>125$ \\
\hline $3 f$ & $>125$ & $>125$ & $>125$ \\
\hline Nevirapine $(\mu \mathrm{g} / \mathrm{ml})$ & $>4$ & 0.075 & $>4$ \\
\hline Lamivudine $(\mu \mathrm{g} / \mathrm{ml})$ & $>20$ & 0.58 & 2.3 \\
\hline Azidothymidine $(\mu \mathrm{g} / \mathrm{ml})$ & $>2$ & 0.0020 & 0,0022 \\
\hline Didanosine $(\mu \mathrm{g} / \mathrm{ml})$ & $>50$ & 18 & 19 \\
\hline
\end{tabular}

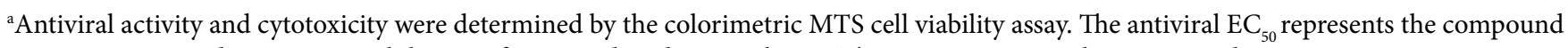
concentration producing $50 \%$ inhibition of virus-induced cytopathicity. The $\mathrm{CC}_{50}$ represents the compound concentration causing $50 \%$ reduction of cell viability. 


\section{ACKNOWLEDGMENTS}

We thank Prof. Lieve Naesens from the Rega Institute for Medical Research, Katholieke Universiteit, Leuven, Belgium

1,3-Tiyazol Halkası Taşıyan Bazı Yeni Siklohekzilidenhidrazit türevlerinin sentezi ve antiviral etkileri

ÖZ

5 Üyeli heterosiklik halka olarak 1,3-tiyazol halkası taşıyan sikloheksilidenhidrazit türevi 6 yeni bileşik sentezlenmiş ve for evaluation of antiviral activity. This work was supported by Istanbul University Scientific Research Project (Project Numbers: BYP-37447 and BYP-48086).

\section{REFERENCES}

1. Rauf A, Farshori NN. Microwave-Induced Synthesis of Aromatic Heterocycles, Springer Dordrecht Heidelberg London New York. 2012.

2. De Souza MVN, De Almeida MV. Drug antiHIV: Past, present and future perspectives. Quim Nova 2003; 26: 366-72.

3. Das J, Chen P, Norris D, Padmanabha R, Lin J, Moquin RV, Shen Z, Cook LS, Doweyko AM, Pitt S, Pang S, Shen DR, Fang Q, De Fex HF, McIntyre KW, Shuster DJ, Gillooly KM, Behnia K, Schieven GL, Wityak J, Barrish JC. 2-Aminothiazole as a novel kinase inhibitor template. Structure-activity relationship studies toward the discovery of $\mathrm{n}$-(2-chloro-6-methylphenyl)2-[[6-[4-(2-hydroxyethyl)-1-piperazinyl)]-2-methyl-4pyrimidinyl]amino)]-1,3-thiazole-5-carboxamide (dasatinib, BMS-354825) as a potent pan-Src kinase inhibitor. J Med Chem 2006; 49: 6819-32.

4. Rehman MZ, Anwar CJ, Ahmad S. An efficient synthesis of 2-alkyl-4-hydroxy-2H-1,2-benzothiazine-3-carboxamide1,1-dioxides. Bull Korean Chem Soc 2005; 26: 1771-5.

5. Knadler MP, Bergstrom RF, Callaghan JT, Rubin A. Nizatidine, an H2-blocker. Its metabolism and disposition in man. Drug Metab Dispos 1986; 14: 175-82.

6. Pasqualotto AC, Thiele KO, Goldani LZ. Novel triazole antifungal drugs: focus on isavuconazole, ravuconazole and albaconazole. Curr Opin Investig Drugs 2010; 11: 165-74.

7. Fox LM, Saravolatz LD. Nitazoxanide: a new thiazolide antiparasitic agent. Clin Infect Dis 2005; 40: 1173-80.

8. Ayati A, Emami S. Asadipour A, Shafiee A and Foroumadi A. Recent applications of 1,3-thiazole core structure in the identification of new lead compounds and drug discovery. Eur J Med Chem 2015; 97: 699-718.

9. Rollas S, Küçükgüzel ŞG. Biological activities of hydrazone derivatives. Molecules 2007; 12: 1910-39.

10. Koruncev D, Babic I, Cvetnic S, Deljac A. Antibacterial and antiviral effects in a series of hydrazides and hydrazones derived from quinoline-2-carboxylic acid. Acta Pharm Jugosl 1975; 25: 241-6.

11. Morgan LR, Thangaraj K, LeBlanc B, Rodgers A, Wolford LT, Hooper CL, Fan D, Jursic BS. Design, synthesis and
CRFK, HeLa, HEL, MDCK, Vero ve MT4 hücre kültürlerinde çeşitli DNA ve RNA virüslerine karşı antiviral aktiviteleri (HIV dahil) incelenmiştir. Bileşiklerin hiçbirinde $100 \mu \mathrm{M}$ 'da DNA ve RNA virüslerine karşı aktivite saptanmamıştır.

Anahtar kelimeler: 1,3-Tiyazol, sikloheksilidenhidrazit, antiviral aktivite

anticancer properties of 4,4'-dihydroxybenzophenone-2,4dinitrophenylhydrazone and analogues. J Med Chem 2003; 46: 4552-63.

12. Ulusoy N. Synthesis and antituberculosis activity of cycloalkylidenehydrazide and 4- aza-1-thiaspiro[4.5]decan3-one derivatives of imidazo[2,1-b]thiazole. Arzneim-Forsch/ Drug Res 2002; 52: 565-71.

13. Koçyiğit-Kaymakçığlu B, Oruç E, Unsalan S, Rollas S. Antituberculosis activity of hydrazones derived from 4-fluorobenzoic acid hydrazide. Med Chem Res 2009; 18: 27786.

14. Riberio IG, Silva KCM, Parrini SC, Miranda ALP, Fraga CAM, Barreiro EJ. Synthesis and antinociceptive properties of structurally planned imidazo[1,2-a]pyridine 3-acylarylhydrazone derivatives. Eur J Med Chem 1998; 33 : 225-35.

15. Prakash D, Prasad SM. Synthesis and antibacterial activities of new quinoline 8-thioglycolylhydrazones. J Indian Chem Soc 1988; 65: 673-5.

16. Johari RB, Nagar R, Sharma RC. Studies on copper (II), nickel (II),cobalt (II) and zinc (II) complexes of acetone salicyloylhydrazone and ethyl methyl ketone salicyloyl hydrazone. Indian J Chem 1987; 26A: 962-3.

17. Krečmerová M, Holý A, Pohl R, Masojidková M, Andrei G, Naesens L, Neyts J, Balzarini J, De clercq E, Snoeck R. Ester prodrugs of cyclic 1-(S)-[3-hydroxy-2-(phosphonomethoxy) propyl]-5-azacytosine: synthesis and antiviral activity. J Med Chem 2007; 50: 5765-72.

18. Ceylan Ş, Bayrak H, Demirbaş A, Ülker S, Alpay-Karaoğlu Ş and Demirbaş N. Synthesis of some new hybride molecules containing several azole moieties and investigation of their biological activities. Russ J Bioorg Chem 2014; 40: 314-29.

19. Koçyiğit-Kaymakçığlu B, Toklu HZ, İkiz S, Bağcıgil AF, Rollas $\mathrm{S}$, Yakut Özgür N and Ak S. Synthesis and antinociceptiveantimicrobial activities of some new amide derivatives of 3,5-di/-and 1,3,5-trimethylpyrazoles. J Enzym Inh Med Chem 2008; 23: 454-61.

20. Tatar E, Küçükgüzel ŞG, Karakuş S, De Clercq E, Andrei G, Snoeck R, Pannecouque C, Öktem Okullu S, Ünübol N, Kocagöz T, Kalaycı S and Şahin F. Synthesis and biological 
evaluation of some new 1,3,4-thiadiazole and 1,2,4-triazole derivatives from $L$-methionine as antituberculosis and antiviral agents. Marmara Pharm J 2015; 19: 88-102.

21. Somogyi L. Notes on the reactions of ketone acylhydrazones under acylation conditions. Tetrahedron 1985; 41: 5187-90.

22. Vicini P, Geronikaki A, Anastasia K, Incerti M and Zani F. Synthesis and antimicrobial activity of novel 2-thiazolylimino5-arylidene-4-thiazolidinones. Bioorg Med Chem 2006; 14: 3859-64.

23. Güzel Ö, İlhan $\mathrm{E}$ and Salman A. Synthesis and antimycobacterial activity of new 2-hydroxy-N-(3-oxo-1- thia-4-azaspiro[4.4]non-4-yl)/(3-oxo-1-thia-4-azaspiro[4.5] dec-4-yl)-2,2-diphenylacetamide derivatives. Monatsch für Chem 2006; 137: 795-801.

24. Göktaş F, Vanderlinden E, Naesens L, Cesur N and Cesur Z. Microwave assisted synthesis and anti-influenza virus activity of 1-adamantyl substituted $\mathrm{N}$-(1-thia-4-azaspiro[4.5]decan4-yl)carboxamide derivatives. Bioorg Med Chem 2012; 20 : 7155-9.

25. SDBS web: http//riodb01.ibase.aist.go.jp/sdbs/cgi-bin/img disp.cgi spectrum no: 3734HSP-44-755 (Accessed: 10 March 2016). 